\title{
Lusioersily
}

\section{Technical Development and Clinical Evaluation of Intelligent Continence Management System at Nursing Home}

Aung, APW., Fook, VFS., Jayachandran, M., Biswas, J., Nugent, CD., Mulvenna, M., Zhang, D., Craig, D., Passmore, P., Lee, JE., \& Yap, P. (2010). Technical Development and Clinical Evaluation of Intelligent Continence Management System at Nursing Home. In Unknown Host Publication (pp. 345-352). IEEE. https://doi.org/10.1109/HEALTH.2010.5556544

Link to publication record in Ulster University Research Portal

\section{Published in:}

Unknown Host Publication

Publication Status:

Published (in print/issue): 01/07/2010

DOI:

10.1109/HEALTH.2010.5556544

\section{Document Version}

Author Accepted version

\section{General rights}

Copyright for the publications made accessible via Ulster University's Research Portal is retained by the author(s) and / or other copyright owners and it is a condition of accessing these publications that users recognise and abide by the legal requirements associated with these rights.

\section{Take down policy}

The Research Portal is Ulster University's institutional repository that provides access to Ulster's research outputs. Every effort has been made to ensure that content in the Research Portal does not infringe any person's rights, or applicable UK laws. If you discover content in the Research Portal that you believe breaches copyright or violates any law, please contact pure-support@ulster.ac.uk. 


\title{
Technical Development and Clinical Evaluation of Intelligent Continence Management System at Nursing Home
}

\author{
Aung Aung Phyo Wai ${ }^{1}$, Foo Siang Fook Victor ${ }^{1}$, Maniyeri Jayachandran ${ }^{1}$, Jit Biswas ${ }^{1}$, Chris Nugent ${ }^{2}$, Maurice \\ Mulvenna $^{2}$, Zhang Daqing ${ }^{3}$, David Craig ${ }^{4}$, Peter Passmore ${ }^{4}$, Lee Jer-En ${ }^{5}$, Philip Yap ${ }^{5}$ \\ ${ }^{1}$ Institute for Infocomm Research, Singapore. \{apwaung, sffoo, mjay, biswas\}@i2r.a-star.edu.sg \\ ${ }^{2}$ University of Ulster, Northern Ireland, UK. \{cd.nugent, md.mulvenna\}@ulster.ac.uk \\ ${ }^{2}$ Institut TELECOM - TELECOM \& Management SudParis, France. daqing.zhang@it-sudparis.eu \\ ${ }^{4}$ Belfast City Hospital, Northern Ireland, UK. \{david.craig, p.passmore\}@qub.ac.uk \\ ${ }^{5}$ Alexandra Hospital, Singapore. Sjer_en_LEE, philip_YAP\}@alexhosp.com.sg
}

\begin{abstract}
Urinary incontinence and diaper use is common among elderly people with dementia staying at nursing homes. Delays in timely diaper change will cause personal, social and economic ramifications to those elderly as well as to the carers who provide nursing care. In order to alleviate these daily care issues, an intelligent continence management system leveraging on sensors, pervasive sensor network, ambient intelligence and reminders is designed and developed. Clinical trial is conducted with multiple elderly people with dementia at a nursing home to evaluate the applicability and usefulness of the developed system. The analysis of trial outcomes and usability studies proves that this will be a feasible and effective approach to tackle the problems faced in managing incontinence effectively at nursing homes.
\end{abstract}

Keywords- urinary incontinence; intelligent continence management system; dementia; elderly; nursing home; ambient intelligence

\section{INTRODUCTION}

Urinary incontinence is highly prevalent among elderly people with dementia at nursing homes $[1,2]$. Incontinence is usually associated with ageing and chronic diseases such as stroke, Alzheimer's disease, Dementia, etc although it is not a normal part of aging. Besides the significant difference in prevalence and incidence of incontinence among various settings, it is commonly regarded as one of the difficult care giving issues in nursing homes [3, 4]. Elderly people with dementia and with irreversible incontinence causes need to wear diapers all the time in order to alleviate the personal, social and clinical ramifications. But the improper management of diapers can still incur setbacks and challenging day to day care issues in long-term nursing care [5]. The elderly may suffer serious health issues due to lack of awareness of soiled diapers and failures in timely diaper change. In order to alleviate this, it is desirable to have an automated system notifying for diaper replacement as soon as incontinence occurs. This can reduce unnecessary visits and diaper checks and at the same time, enable immediate remedy with proper assessment and diagnosis [6].
Although incontinence is not a direct cause for functional decline or death, it will be an indicator of frailty and poor care giving [7]. There are different treatments available such as behavior therapy, pharmacological and use of absorbent pads $[1,8]$. But irreversible causes of incontinence with decline in chronic health conditions are still a problem at nursing homes [6]. Especially in nursing home settings, proper assessment and management of incontinence is very labor intensive and challenging issue. If we study the different incontinence care strategies at nursing homes, the needs of collaboration and integration among different approaches and relevant parities can be clearly seen [5].

With advances in Information and Communication Technology (ICT), the real and utmost needs of frail elderly can be fulfilled to some extend through anyplace and anytime monitoring and coaching system [9, 10]. Incontinence being one of the major geriatric syndromes at nursing homes, different technical solutions are proposed, from simple sensor design to complex management system. The use of temperature sensors to detect and record incontinence events can be found in $[11,12]$. In [13] urine collecting device based on space technologies is designed and developed specially for incontinent disabled women. The monitoring system leveraging on sensor network and RFID technologies is also proposed to detect incontinence episodes [14]. Our solution encompasses the full spectrum of managing incontinence operations from wetness sensing, distributed monitoring and reminding incontinence events to assisting nurses for timely diaper change. This can be realized through different enabling technologies such as pervasive sensor network [15], Ambient Intelligence [16] and audio/video reminders [17]. The evaluation trial was conducted with multiple elderly people with dementia at a nursing home after deploying the intelligent Continence Management System (iCMS).

This paper presents technical development and clinical evaluation of iCMS with multiple elderly people with dementia at a nursing home. Section II presents objectives, consideration and issues pertaining to development of iCMS. The extended technical design and development will be discussed in section 
III. Then, clinical trail planning and evaluation of iCMS at nursing home will be explained in section IV. Finally, analysis outcomes of clinical trial results and possible future works will be explored in section $\mathrm{V}$.

\section{Intelligent ContinenCE MANAGEMENT System}

According to initial requirement analysis and feasibility studies, a tiered system architecture was designed and software components were developed to implement the system [18]. The main goal of iCMS is to enable timely diaper change through smart sensing over wireless sensor network and context-aware reminders. As shown in Fig. 1, iCMS can be implemented as a 3-tier architecture that consists of two main components: sensing network system and intelligence system.

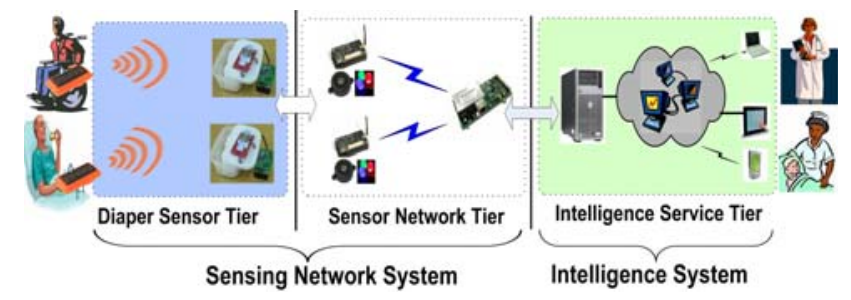

Figure 1. iCMS: 3-Tiered Architecture with 2 main sub-systems

Sensing network system supports prompt detection of wetness episodes with embedded sensors and anywhere anytime observation through underlying pervasive sensor network. Intelligence system handles management of whole system operations and the function of reminding carers through different alerts/reminders. So, iCMS can provide assistance to carers in effective management of incontinence without extra efforts while achieving desirable incontinence care. This will promote quality of life of those incontinent elderly as well as improve the quality of care at nursing homes.

\section{A. Clinical Issues and Requirements}

In order to use assistive system like iCMS in day to day care operations, clinicians demand simplicity, reliability and user-friendly aspects from such system. Effective collaboration and seamless integration of iCMS into specific care settings are also important in order to have desirable level of continence care and diagnosis [5, 19]. The problem of incontinence at nursing homes is due to difficulties in providing personalized care to the residents. There is limited resource in terms of nursing staff to support multiple residents with varying incontinence needs [20]. The proposed solution must capture these requirements and fulfill the needs of patients as well as carers with minimal disturbance to their daily routine and operations. The solution should be easily adaptable and should be inline with current nursing care practices at the particular nursing home. Fig. 2 provides an overview of how different levels of nursing staff (senior and junior nurses) involved in diaper replacement interact with iCMS. In accordance with the care model at nursing home, iCMS operation has to be suitably modified to satisfy the needs of all nursing staff without overloading or disturbing their primary care giving tasks.

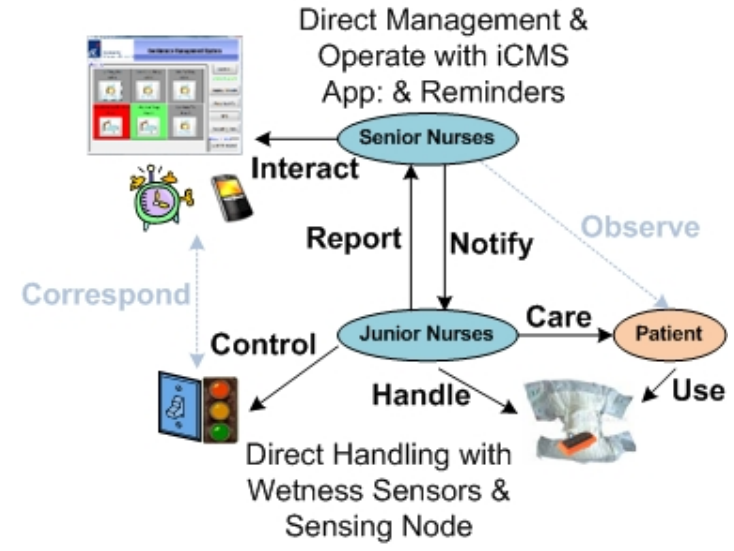

Figure 2. Nursing Home Care Scenarios and Interaction with iCMS

According to Fig. 2, the senior nurses directly use iCMS through mobile phones and management console at the nursing station. Upon receiving the wetness alerts, they instruct nearby on-duty junior nurses to attend and provide care to particular elderly. Then, the junior nurses will perform actual diaper replacement and manage iCMS's operations through "control button" next to patient's bed. Overall, the desirable features from clinical perspective can range from a) unobtrusive continuous monitoring, b) context-aware prompting to c) personalized assessment and, d) preventive measures (e.g, time voiding, continence planning).

\section{B. Technical Challenges and Limitations}

Although various technical considerations were taken care of in the initial design of iCMS, limitations and new requirements of iCMS were identified from initial pilot study at nursing home [21]. From that study, we gained insights on the aspects of iCMS that have to be revised in order to provide desirable assistance. We also learned that daily operational issues such as forgetting to put sensor inside diaper, placing wetness sensor in wrong orientation, etc will hinder the real capabilities of iCMS. Some parameters including usability, acceptance, sensor network lifetime, system performance, etc. are necessary to investigate their effects in providing assistance to multiple patients [22]. In order to gain acceptance from clinicians, every incontinence episode must be reliably detected anywhere, anytime to enable effective continence management at nursing home. Although current sensor has small form factor, it is not small enough and importantly, is nondisposable. Moreover, the reliability and robustness issues of underlying sensor network still have rooms for improvement in areas such as energy efficiency, in-network processing, location tracking, etc.

\section{EXTENDED System DESIGN AND DEVELOPMENT}

Based on experiences and lessons learned from the initial clinical trial with iCMS [18, 21], the following feature enhancements and functional extensions are incorporated into the system. 


\section{A. Extended Sensing of Patient's Contexts}

In order to enable continuous diaper monitoring, based on the presence of the patient, additional sensing modalities are integrated into sensing node. By integrating pressure sensors (Force Sensing Resistor strip, Interlink Electronics, Camarillo $\mathrm{Ca}, \mathrm{USA}$ ) and motion/orientation sensors (Accelerometer breakout, SparkFun Electronics, Boulder Co, USA) to patient's bed and wheelchair as shown in Fig. 3, the contexts specific to particular patient can be easily determined. The resulting context-aware sensing infrastructure enabled us to decide on the sensors that need to be turned on, selecting the appropriate sensing node based on patient specific context information. This will also help to conserve the battery power. If there is no patient on wheelchair, the sensors and associated components can go to sleep mode. From day to day operation, it can avoid the extra workloads of switching the system on and off manually. In addition, the context information also provides the ability to capture daily personal profile of the subjects.

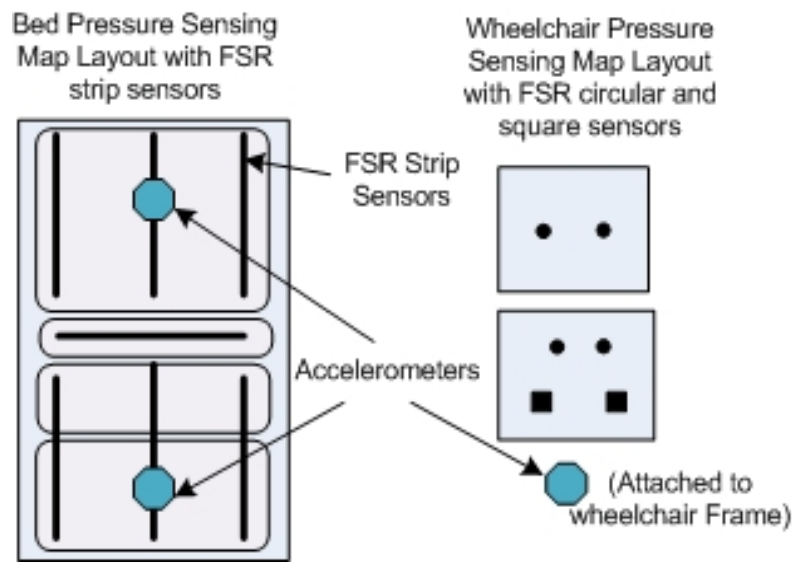

Figure 3. Pressure and motion sensors integrated into bed and wheelchair

\section{B. Improved System Design and Functionalities}

Considering simplicity, extensibility and efficiency, functionalities of different components of iCMS are redesigned and implemented to cover the drawbacks and limitations faced in initial pilot study. The new functional components that support the desired sensing, reminder system and features for intelligence are developed according to a 3-tiered architecture. As shown in Fig. 4, seven different software and hardware components are now involved in the iCMS design. Same as before, Wetness Alert Diaper (WAD) includes the diaper with wireless wetness sensor that is attached to the subject. Elderly Occupancy Detector (EOD) monitors the presence, location and movement of subject as it is integrated into patient's bed and wheelchair. Then, carers are notified about the occurrences of incontinence episodes through Events \& Activity Reminder (EAR), Nurse Mobile Phone (NMP), Incontinence Episode Visualizer (IEV) and Interactive Control Switch (ICS). Upon receiving reminders from any of these components, carers attend to the patient and perform necessary care giving and manage the system operations through either IEV or ICS. As its name implies, Intelligence Management Server (IMS) manages the whole system operations and collects data and events occurred during the operation of the system.

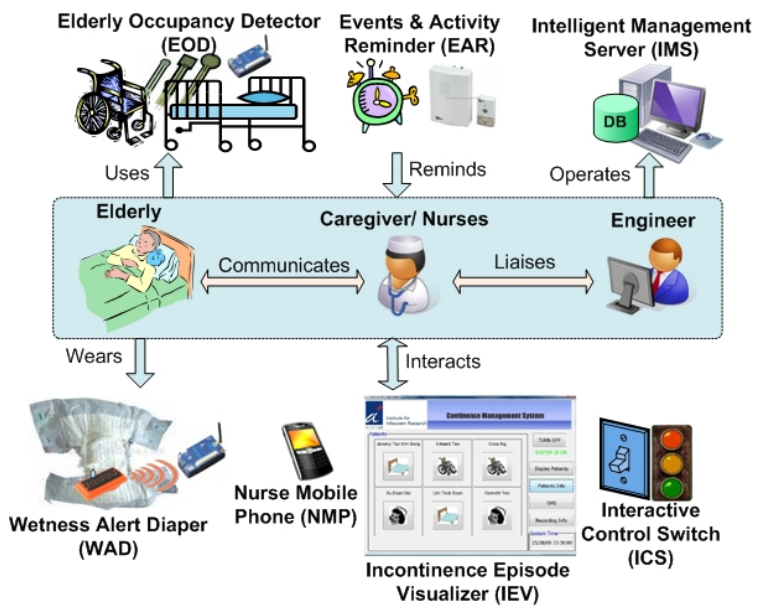

Figure 4. Improved iCMS Functional Components

\section{Revised Usability and Operation Scenarios}

Although the operation guidelines and user manuals about the system are provided, there were still day to day operational problems in our pilot study. So usability survey and focus discussion are performed again with the help of nurses in order to design an easy-to-use system for operations at nursing home. Based on our analysis, the system operations are revised to simplify the interaction and operations among different entities as shown in Fig. 5. Firstly, the wetness events and change in patient's contexts are detected and notified through multi-hop sensor network. With provided intelligence services, appropriate reminders are sent to carers to inform the detected incontinence episodes. Finally, the carers provide necessary care to the needy elderly and interact with system states. Intelligence server operates according to detected events and carer's interactions and records those data in centralized database.

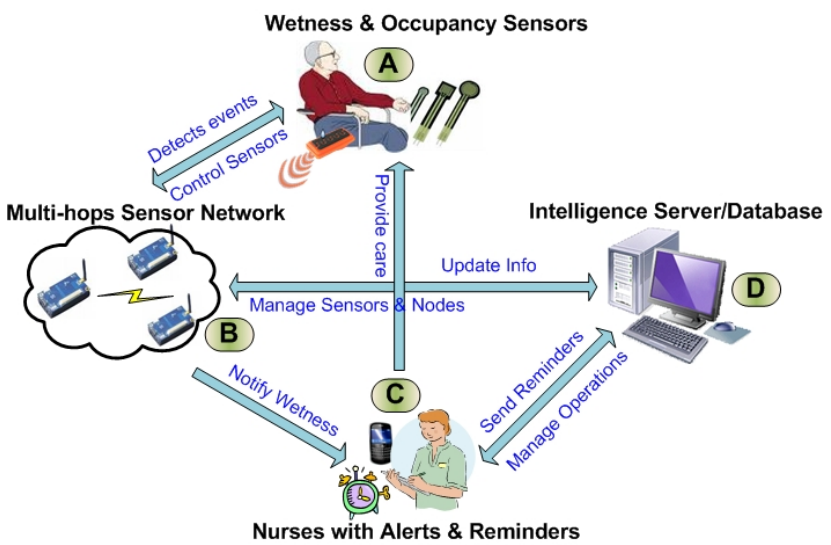

Figure 5. iCMS System Operation Overview

From evaluation trial with initial prototype, carers expressed that it is not intuitive to understand different states that belongs to particular subjects as well as in managing 
multiple subjects with the provided console applications [21]. Thus, current iCMS's application front end (IEV) was revamped with a completely new user-friendly outlook to display patient incontinence states more intuitively as shown in Fig. 6. This permits user-friendly information display as well as easy management of multiple subjects simultaneously.

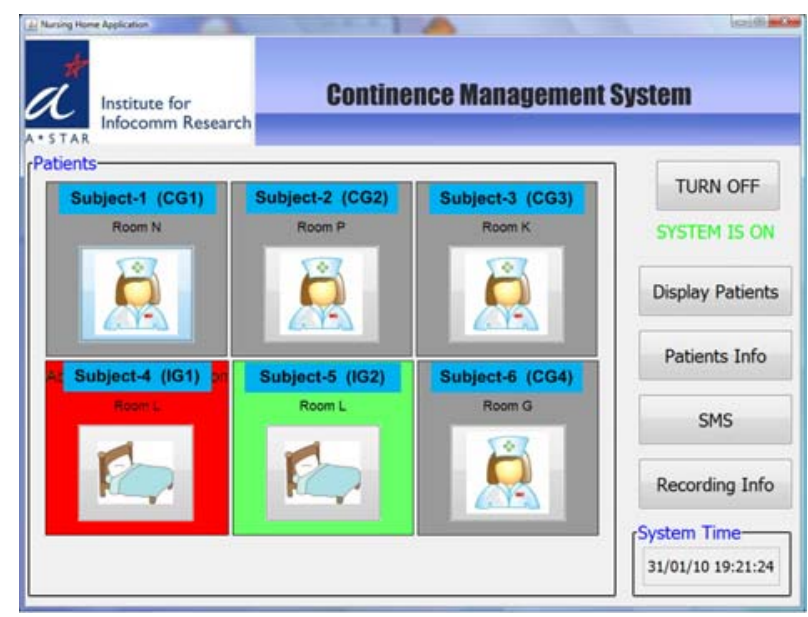

Figure 6. Screenshot of iCMS main application management interface

The soiled status indication about the patient's diaper is provided through flashing visual as well as audible alerts from IEV (Fig. 6). So, the carers could manage the system operations (start/stop monitoring) from either IEV or by means of control button at ICS near the patient's bed. For ground truth recording of all incontinence states, only manual bladder charting was used. So we developed an application with intuitive user interface to record the patient's incontinence related information as shown in Fig. 7. Also, in manual bladder charting forms, cue words such as Wet/Dry/NA (wetness status), No/Small/Half/Full (diaper wetness level) are provided instead of open-format writing used previously.

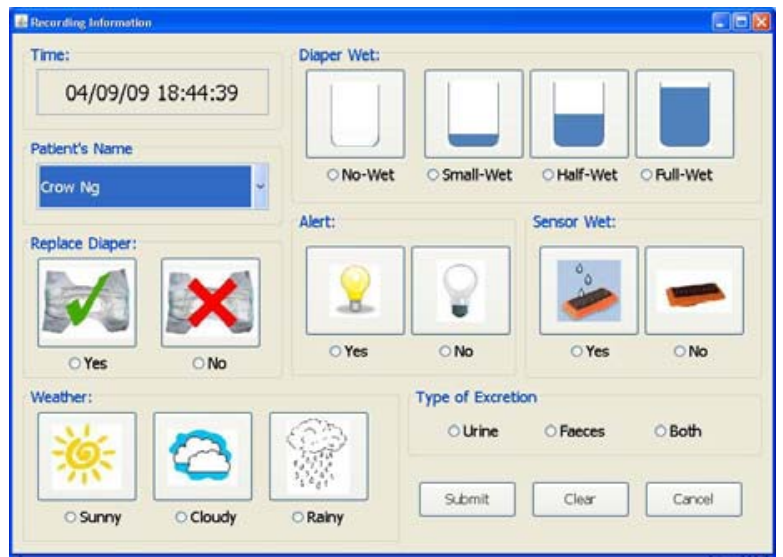

Figure 7. Snapshot of Incontinence status recording user interface

As mentioned before, simple control button and light indicators, ICS, are provided next to the patient in order to assist carers for easy operation of the system according to the patient's incontinence and care giving status. Fig. 8 shows different instances of light indicators according to system operation, detected incontinence states and user interactions.
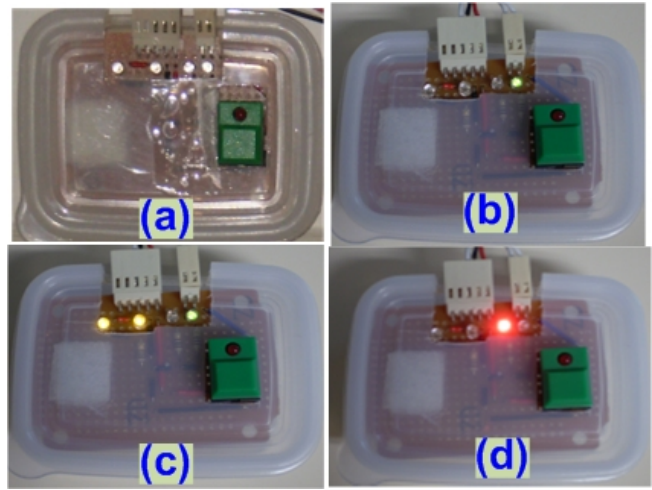

Figure 8. Light Indicators with respect to iCMS states: (a) No Monitoring (b) Monitoring (c) Wetness Detection (d) System Sleep (Diaper Replacement)

\section{Hardware and Software Development}

According to improved system design and functionalities, shown in Fig. 4, the hardware and software components are implemented as two major subsystems. Currently, sensing network system consists of five different types of wireless nodes: sensing (fixed versus mobile), relay/alert, attention and gateway nodes. The sensing node was completely redesigned and implemented to capture different sensor states, as well as to detect the malfunction of wetness sensor. Generally, the fixed nodes are installed nearby the patient's bed whereas the mobile nodes are attached to patient's wheelchair. Attention node is deployed at the table next to patient's bed for logging the frequency and timing of carer's attendance to particular patient. No important modifications are made to both relay/alert and gateway nodes. In current setup, intelligence system physically consists of a computer where sensor network gateway node with Ethernet base-station and SMS gateway are connected. Besides revamped user interface design, logging of network events or state changes and users interactions with IEV and ICS are also integrated into current implementation.
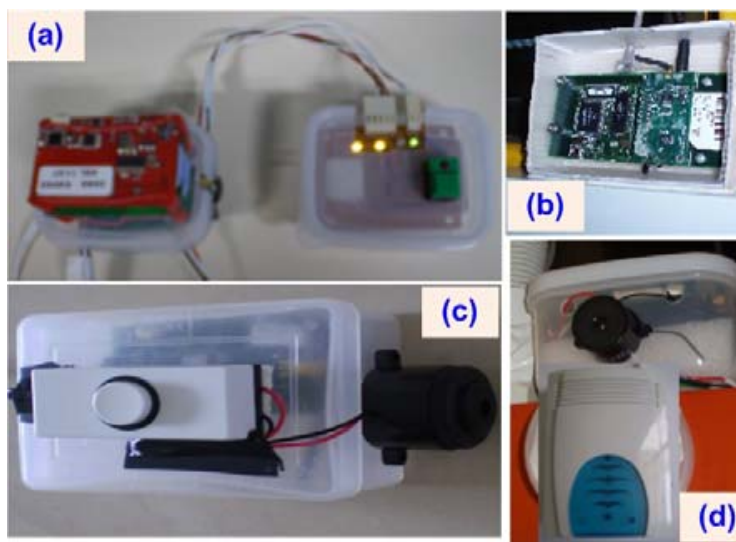

(b)

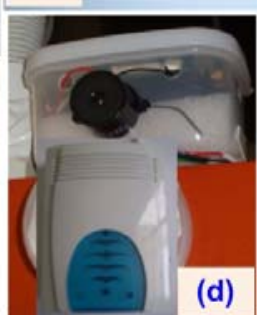

Figure 9. Different sensing network system components (a) Sensing Node (b) Gateway Node (c) Attention Node (d) Alert/Relay Node 


\section{Clinical Design, Deployment And Evaluation}

In order to conduct proper clinical trials with multiple subjects at nursing home, specific trial design, protocols and evaluation criteria are identified to validate the performance as well as usability of iCMS.

\section{A. Trial Design \& Protocols}

As discussed, formal system deployment scenarios and trial design processes are designed and developed from technical as well as clinical perspectives. From the pilot study we learnt that user errors and complexity in operation flow results in low efficiency and sometimes, makes the system useless. The inclusion and exclusion criteria considered in recruiting subjects are defined (Table I) according to inputs from medical doctors and nurses. Then, potential elderly subjects who meet required criteria are identified to be included in the evaluation trial. Among them, a group of elderly (6-10) are finally recruited after obtaining consents from them or their families.

\section{TABLE I. CRITERIA CONSIDERED FOR RECRUITING TRIAL SUBJeCtS}

\begin{tabular}{|l|l|}
\hline \multicolumn{1}{|c|}{ Inclusion Criteria } & \multicolumn{1}{|c|}{ Exclusion Criteria } \\
\hline Physical Disability & $\begin{array}{l}\text { Acutely ill with unstable } \\
\text { vital parameters }\end{array}$ \\
\hline Suffer from Dementia & $\begin{array}{l}\text { Having pacemakers and } \\
\text { implantable defibrillators }\end{array}$ \\
\hline $\begin{array}{l}\text { Having incontinence } \\
\text { and wearing diaper }\end{array}$ & Under special medications \\
\hline
\end{tabular}

In current trial, the recruited subjects can be divided into intervention group (IG) and control group (CG). This is meant to fully evaluate and cross-validate the system performance. The purpose and operational differences between these two subject groups involved in the trial are described in Table II. The main aim is to measure whether benefit in IG compared to routine diaper checks performed in CG. Also, basic trial protocols with respect to identified trial subject groups were designed to effectively carry out daily care operations and validate the system's performance.

TABLE II. CATEGORIES OF HUMAN SUBJECTS INVOLVED IN TRIAL

\begin{tabular}{|c|l|l|}
\hline Patient & \multicolumn{1}{|c|}{ Purpose } & \multicolumn{1}{|c|}{ Deployment } \\
\hline IG & $\begin{array}{l}\text { Wetness sensor is inserted } \\
\text { into diaper. Soiled diaper is } \\
\text { observed through iCMS }\end{array}$ & $\begin{array}{l}\text { Sensing node is } \\
\text { deployed nearby } \\
\text { patient. }\end{array}$ \\
\hline CG & $\begin{array}{l}\text { No wetness sensor is used. } \\
\text { Only manual scheduled } \\
\text { diaper check is performed. }\end{array}$ & $\begin{array}{l}\text { Attention node is } \\
\text { placed next to } \\
\text { patient's bed. }\end{array}$ \\
\hline
\end{tabular}

As shown in Fig. 10, the numbers mentioned in each block represent the planned and actual subjects involved in current clinical trial. Due to selection criteria (Table II) and availability of subject, the actual subjects involved in trial are only 6 instead of 10. Initially, we planned to divide the subjects into 3 IG and 3 CG with a mixture of male and female subjects. Before starting the trial, one of the female IG subjects was excluded due to the use of catheter. So a total of 2 male IG, 2 female $\mathrm{CG}$ and 2 male $\mathrm{CG}$ are involved in current one month evaluation trial.

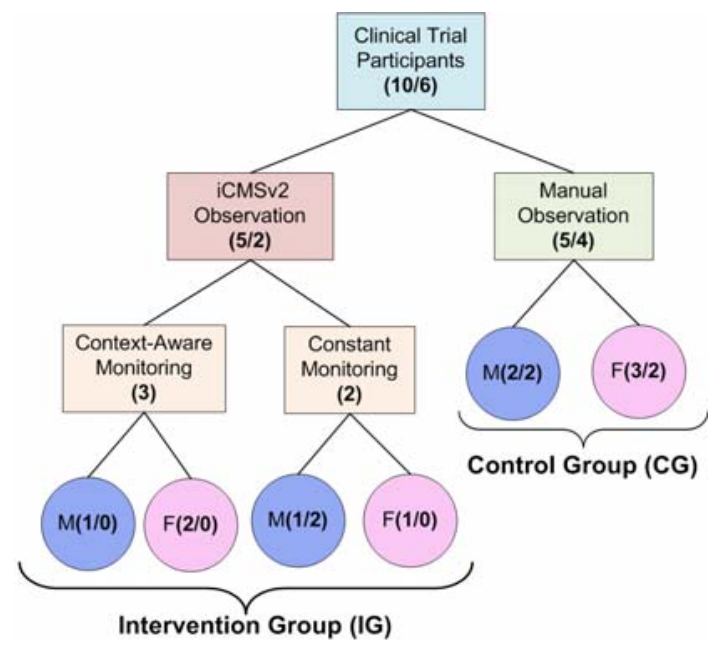

Figure 10. Incontinence Subjects recruited for nursing home trial

The basic demographic profile of all trial subjects can be found in Table III. Based on observation throughout the trial, they mostly lie in the bed and go out of the bed only once or twice a day using reclined wheelchair. As mobile sensing node is attached to the wheelchair, incontinence episodes monitoring can be performed continuously as long as the patient stays within the coverage range ( 5 to $10 \mathrm{~m}$ ) of sensing node.

TABLE III. DEMOGRAPHIC PROFILE OF SUBJECTS INVOLVED IN TRIAL

\begin{tabular}{|l|l|l|l|}
\hline $\begin{array}{l}\text { Subje } \\
\text { ct }\end{array}$ & Age & Gender & Diseases \\
\hline IG1 & 73 & Male & $\begin{array}{l}\text { Stroke, Renal Impairment, IHD, } \\
\text { Hypertension }\end{array}$ \\
\hline IG2 & 50 & Male & $\begin{array}{l}\text { Advanced Neurodegenerative } \\
\text { disease }\end{array}$ \\
\hline CG1 & 58 & Male & $\begin{array}{l}\text { Cerebrovascular Disease(stroke), } \\
\text { Hypertension }\end{array}$ \\
\hline CG2 & 81 & Female & Parkinson's Disease, Dementia \\
\hline CG3 & 76 & Female & Hyperlipidaemia, Hypertension \\
\hline CG4 & 63 & Female & $\begin{array}{l}\text { Chronic Schizophrenia, Hyperli- } \\
\text { pidaemia, Parkinson's Disease }\end{array}$ \\
\hline
\end{tabular}

\section{B. Nursing Home Trial Deployment}

All software and hardware components are deployed at nursing home considering the locations of different patients. The sensing and attention nodes are deployed near the bed and at wheelchair allocated to individual subjects. The locations of relay/alert nodes are identified based on the results of bidirectional wireless communication tests during deployment. Then, audible reminders are integrated into relay/alert nodes deployed at hallway to notify the nurses of detected wetness 
events. Fig. 11 shows settings of different hardware, including a computer at nursing station, deployed sensor network system, operational manuals, sensors and pouches used in trial.
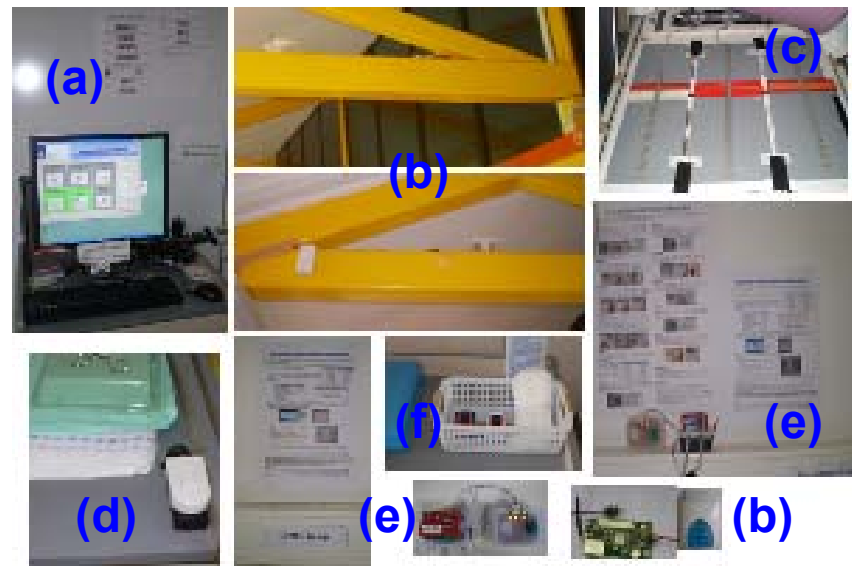

Figure 11. Nursing Home Deployment of iCMS Components (a) iCMS application@nurstion station (b) relay/alert nodes (c) pressure sensing map

(d) attention node (e) sensing node (f) wetness sensors with pouches

\section{Trial Operations, Results and Analysis}

Along with improved trial design and protocols, extensive manual diaper checks are scheduled, to check every two hours instead of normal 4 or 5 times a day as shown in Table IV. This will enable us to correctly validate the performance and usefulness of iCMS as well as applicability of extensive diaper checks. After operating iCMS under daily care operation over one month period, a preliminary evaluation analysis is performed to understand the system capabilities as well as associated clinical gains.

TABLE IV. DIFFERENT MANUAL SCHEDULED DiAPER CHECKS: EXTENSIVE AND NORMAL INTERVALS

\begin{tabular}{|c|c|c|c|}
\hline \multicolumn{2}{|c|}{ Day Schedule } & \multicolumn{2}{|c|}{ Night Schedule } \\
\hline Extensive & Normal & Extensive & Normal \\
\hline 7:00 am & \multirow{2}{*}{ 8:00 am* } & $7: 00 \mathrm{pm}$ & \multirow{2}{*}{$7: 30 \mathrm{pm}^{*}$} \\
\hline 9:00 am & & 9:00 pm & \\
\hline 11:00 am & \multirow{3}{*}{$12: 30 \mathrm{pm}^{*}$} & $11 \cdot 00 \mathrm{~nm}$ & $10: 00 \mathrm{pm}^{\#}$ \\
\hline \multirow{2}{*}{$1: 00 \mathrm{pm}$} & & $1: 00 \mathrm{am}$ & \multirow{3}{*}{ 2:00 am* } \\
\hline & & \multirow{2}{*}{ 3:00 am } & \\
\hline $3: 00 \mathrm{pm}$ & \multirow{2}{*}{$4: 00 \mathrm{pm}^{\#}$} & & \\
\hline $5: 00 \mathrm{pm}$ & & $5: 00 \mathrm{am}$ & $4: 00 \mathrm{am}^{\#}$ \\
\hline
\end{tabular}

According to preliminary analysis done with trial results, we realized that the scheduled two hourly checks, although good in theory, are very difficult to carry out in practice. From manual incontinence documentation, the average frequency of diaper checks or recordings is always lower than planned check. Although the manual diaper checks are performed only $59 \%$ of the times scheduled (Table IV), they over-performed on the average of two times more than the actual diaper replacement frequencies. The preliminary analysis outcomes from current trials show an average of $75 \%$ sensitivity and $96 \%$ specificity in detection of soiled diapers with actual diaper replacement. Table $\mathrm{V}$ shows the sensitivity and specificity outcomes achieved with two IG patients. This shows there are several non-functional and usability factors that affects system operations although the same carers provide care to the patients. In current deployment, these two IG patients are located next to each other. Moreover, they received the same care procedure and operation during the trial.

TABLE V. Performance analysis of Detecting Solled Diapers

\begin{tabular}{|c|c|c|}
\hline Patient & Sensitivity & Specificity \\
\hline IG1 & $69 \%$ & $95 \%$ \\
\hline IG2 & $80 \%$ & $97 \%$ \\
\hline
\end{tabular}

As shown in Fig. 12, the trial outcomes indicated that wetness detection from iCMS with $97 \%$ specificity is comparable with actual diaper replacement frequencies. On the other hand, both planned and actual scheduled diaper checks resulted in unnecessary attendance to the subject and diaper checking as shown in Fig. 12.

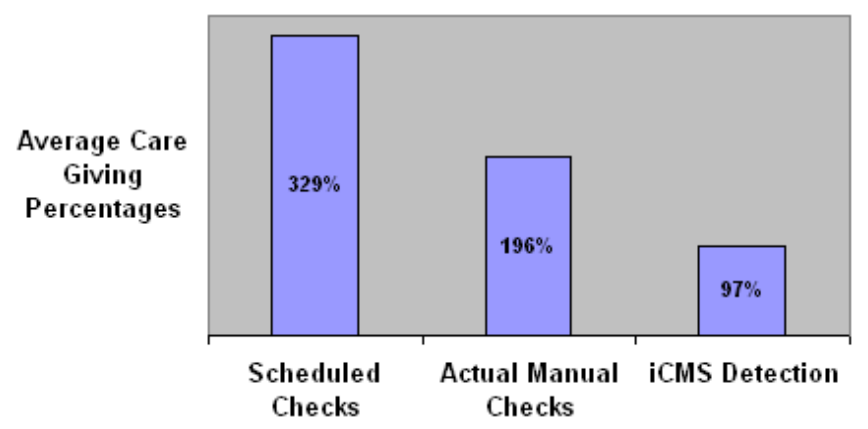

Figure 12. Comparison of different diaper wetness checking and detection frequencies

It is also important to observe location of wetness inside the diaper as well as the level of wetness in order to place wetness sensor inside diaper. These parameters determine the proper attachment of wetness sensor to detect all wetness episodes. Fig. 13 shows the statistics of average diaper wetness levels recorded from all trial subjects.

\section{Statistics of Different Diaper Conditions}

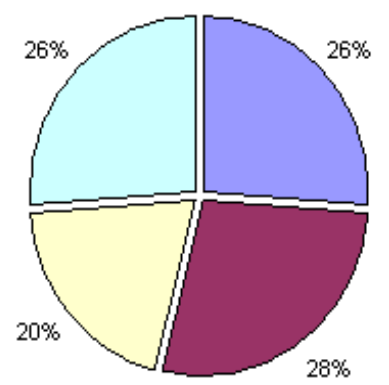

口Full Wetness

aHalf Wetness

口Small Wetness

口No Wetness

Figure 13. Comparison of different levels of wetness observed manually 
But the identification of precise location (front, bottom, back, all, none) and level (none, little, half, full) of wetness in diaper is generally subjective. Due to the importance of proper sensor placement with current sensor design, those information are obtained through manual recordings by the carers. We also observed that sometimes the diaper may be changed although the diaper is not fully wet. It completely depends on carer's discretion as to whether it is necessary to change the diaper, based on his or her observation of diaper wetness. Although the carers usually place wetness sensor near the middle of the diaper, it can detect generally well in full and half wetness conditions regardless of position of wetness occurrences inside the diaper. During the trial, the functionality of audible reminders malfunctioned once due to missed acknowledgement packet in the network. According to feedbacks provided by carers in incontinence documentation and iCMS logs, we learnt that the light indicators from ICS (Fig. 8) did not display correctly in few occasions because of operator error (human error). Under correct operation, carers have to press the button at ICS twice when WAD detects wetness and then, perform diaper replacement. The first press is to disable the reminder and sensors, while diaper change was being carried out. Then, second press is required to re-enable the sensing function so that iCMS could resume monitoring. However on more than one occasion, the button was not pressed properly, leaving the display state at ICS showing the alert or disable states, even after the incontinence episodes had been properly attended to. Noticeably though, such malfunctions only affected the outer (display) layer of the system since the system internally had an auto-reset feature based on a time-out, that took care of such human errors.

\section{Statistics of Diaper Wetness Detection Locations}

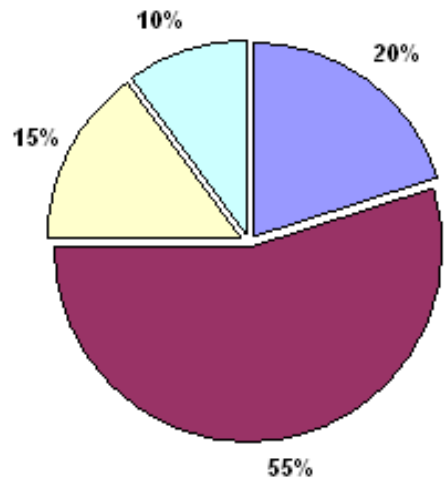

\section{口Front of Diaper}

aMiddle of Diaper

口Eack of Diaper

口Others (side areas, all areas, etc)

Figure 14. Different diaper wetness locations through manual observations

From data collected at the attention nodes deployed next to the CG subjects, only $50 \%$ of the scheduled diaper checks were actually carried out. The possible reasons include carer forgetting to press the button; carer not attending to the patient according to schedule and wireless message losses. Further analysis of collected data is still in progress in order to determine the limitations and to refine the parameters that affect the performance of iCMS. Although we deployed and collected data from EOD, their contexts are not yet considered to provide reminders and to determine wetness events. Incorporation of such context information is currently in progress. The system operation logs also indicated that there is no usage of IEV in diaper management as well as incontinence recording. The reason is that after the introduction of the ICS near patient's bed, user interface at IEV is no longer necessary. The carers found it more convenient and preferred to control system operations from the bedside rather than from the centralized application at nursing station.

\section{DISCUSSION AND FUTURE WORKS}

Although current extensions address some of the drawbacks and limitations faced in the initial prototype, there are still open issues and challenges from technical as well as clinical perspectives. Some requirements and trial data are still being examined to assess their influence and performance over the long-term. Due to the involvement of battery-powered wireless sensor nodes with mobility and ad-hoc networking paradigm, the reliability of message exchange, event notification and reminder actuation are apparent challenges faced in real deployment. Another challenge is to evaluate the system's efficiency and effectiveness correctly in managing incontinent patients. Currently, determining reliable ground truth in real care settings still remains as a challenging issue.

The underlying non-functional and usability issues that affect the operations of iCMS should be identified and studied in future. Reference [20] pointed out areas that affect the acceptance and use of assistive devices available for managing incontinence. Along with this, similar study should be carried out with iCMS to identify the impact and perception of its use, considering the parameters described in Fig. 15. We also realized that sometimes the manual recordings had inconsistent information in them. For example, full diaper wetness was recorded; however the diaper was not replaced. Since the manual record is taken as the source for providing ground truth in the trial, these errors can lead to improper system evaluation. The day to day problems pertaining to proper handling of system will need to be studied thoroughly to design more user friendly and personalized continence management solutions. It is essential to establish ground truth validity by identifying possible causes of errors that possibly degrade actual effectiveness of the system. Moreover, the carers may be easily distracted with their busy schedule to attend to scheduled diaper checks or to respond to prompted reminder events. It is important to attract attention of the carers through pervasive and intuitive means without causing annoyance.

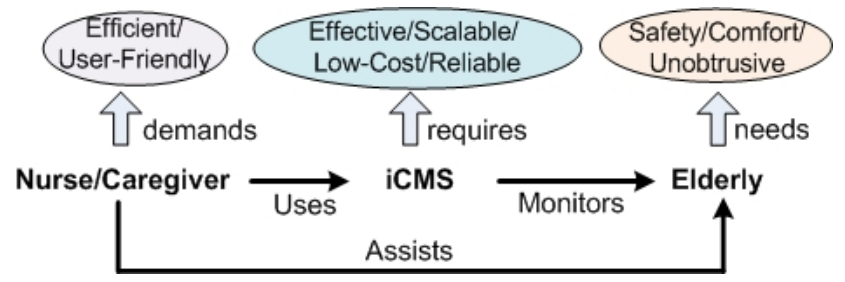

Figure 15. Parameters to evaluate the continence management using iCMS

According to [6], iCMS is currently meant only to sustain or eliminate setbacks in managing diapers but it lacks in active continence promotion to improve incontinence care. So, active cooperation and integration among nursing staff and iCMS will only enable to promote and achieve good continence care practices at nursing home [23]. The next important step will 
involve evaluation of the fully functional system, exploiting context-aware intelligence and usability issues. This includes incorporating additional functionalities and features such as context-aware reminders and continence planning. Then, a clinical trial with larger sample size using cross-over of different patient groups will need to be conducted. Several trials considering technical, clinical and usability aspects will be carried out iteratively, making improvements with each step, till we reach our goal of promoting quality of life and improving quality of incontinence care at nursing home.

\section{CONCLUSION}

Elderly people with dementia who suffer from incontinence will suffer serious social, personal and medical ramifications if the proper management and diagnosis is not in place. For those with prevalent diaper use, timely diaper change is necessary to properly manage incontinence. In order to provide assistance in timely diaper change at nursing homes, iCMS is proposed. Furthermore, technical development and clinical trial studies are also conducted to validate effectiveness and applicability of iCMS. The trial outcomes from multiple elderly people at nursing homes illustrate high sensitivity with respect to ground truth data. Timely diaper change is feasible with iCMS upon occurrences of incontinence episodes. From this study, we are planning to enhance and extend the current functionalities of iCMS further, aiming to achieve desirable incontinence care at nursing homes.

\section{ACKNOWLEDGMENT}

This work has been partially funded by American Alzheimer's Association through Everyday Technology for Alzheimer's Care grant no. ETAC-07-59979. We would like to extend our acknowledgements to nursing staff, carers and patients from local nursing home who participated in various questionnaires and clinical trials.

\section{REFERENCES}

[1] Conner E.L and Lind L., "Urinary incontinence in nursing homes: epidemiology and management guidelines", Primary Care Update for OB/GYNS, Vol. 8(6), pp. 248-252(5), Nov 2001.

[2] Saxer S., Halfens RJ, Muller M and Dassen T, "Risk factors for unrinary incontinence in nursing home residents" , Swiss Med Wkly, Vol. 135(33-34), pp.495-502, Aug 2005.

[3] S.M. Peet, C.M. Castleden, C.W.McGrother and H.M.Duffin, "The management of urinary incontinence in residential and nursing homes for older people", Age and Ageing, Vol. 25, pp.139-143, 1996.

[4] Victor W Nitti, "The Prevalence of Urinary Incontinence", Reviews in Urology, Vol. 3(Suppl 1), pp. S2-S6, 2001.

[5] Newman DK, Fader M and Bliss DZ, "Managing Incontinence using technology, devices and products: directions for research", Nursing Research, Vol. 53(6-Supp), pp. S42-S48, 2004
[6] Mary H. Palmer, "Urinary incontinence quality improvement in nursing homes: where have we been? where are we going?", Urologic Nursing, Vol. 28(6), pp. 439-444, 2008.

[7] Holroyd-Leduc JM, Mehta KM and Covinsky KE, "Urinary Incontinence and its association with death, nursing home admission, and functional decline", Journal of American Geriatic Society, Vol. 52(5), pp. 712-718, May 2004.

[8] J. Durrant and J. Snape, "Urinary Incontinence in nursing homes for older people," Age and Ageing, Volg. (32), pp.12-18, 2003.

[9] Nugent, C.D, "ICT in the elderly and dementia", Aging \& Mental Health, Vol. 11(5), pp.473-476, 2007.

[10] T. Hori et al, "Sensor Network for Supporting Elderly Care Home", Proc. of IEEE Sensors, Vol. 2, pp. 575-578, Oct 2004.

[11] Eckford S.D, Finney R., Jackson S. R. and Abrams P., "Detection of urinary incontinence during ambulatory monitoring of bladder function by a temperature sensitive device", British Journal of Urology, Vol. 77(2), pp. 194-197, 1996

[12] Cusick G et al, "A system for logging incontinence events using a simple disposable sensor", Journal of Engineering in Medicine, Vol. 217(4), pp. 305-310, 2003.

[13] E. Tinnion and F. Jowitt, "The active urine collection device: a novel continence management system focusing particularly on the needs of disabled women", Disability and Rehabilitation, Vol. 22(16), pp. 745$748,2000$.

[14] Ang. L. M et al, "Wireless intelligent incontinence management system using smart diapers", Proc. of $5^{\text {th }}$ ECTI-CON, pp.69-72, 2008.

[15] Frank L. Lewis, “ Wireless Sensor Network,” in Smart Environments: Technology, Protocols and Applications(Wiley Series on Parallel and Distributed Computing), D. J. Cook and S.K. Das, Ed. WileyInterscience, 2004, pp.13-46.

[16] D.J. Cook, J.C. Augusto and V.R. Jakkula, "Ambient Intelligence: Technologies, appications and opportunities", Pervasive and Mobile Computing, Vol. (5), pp. 277-298, 2009.

[17] Veldhoven, E. R, Vastenburg, M. H. and Keyson, D. V.," Designing an Interactive Messaging and Reminder Display for Elderly", Proc. of the European Conf. On Ambient Intelligence, LNCS, Vol. 5355, pp. 126140, 2008.

[18] J. Biswas et al, "Design of a smart continence management system based on initial user requirement assessment", Proc. of ICOST, LNCS, Vo. 5210, pp.62-72, Jun-Jul 2008.

[19] Marilyn J. Rantz et al, "A technology and nursing collaboration to help older adults age in place", Nursing Outlook, Vol. 53(1), pp. 40-45, 2005.

[20] McDonagh D, Reid A and Torrens G, "Satisfying the real needs of the individual: Living and thriving with urinary continence", Incontinence: the Engineering Challenge. Seminar organised by the Medical Engineering Division. 29-30 Oct 2003.

[21] Aung A. P. W. et al, "Smart wireless continence management system for persons with dementia", Journal of Telemedicine and e-Health, Vol.14(8), pp.825-832, 2008.

[22] R. Steele, A. Lo, C. Secombe and Y.K. Wong, "Elderly person's perception and acceptance of using wireless sensor networks to assist healthcare," International Journal of Medical Informatics, Vol. 78(12), pp. 788-801, 2009

[23] Jayna M. Holroyd-Leduc, Courtney H. Lyder and Cara Tannenbaum, "Practical management of urinary incontinence in the long-term care setting”, Annals of Long-Term Care, Vol. 14(2), Feb 2006. 